\title{
Article \\ Relaxed Modulus-Based Matrix Splitting Methods for the Linear Complementarity Problem ${ }^{\dagger}$
}

\author{
Shiliang Wu ${ }^{1}$ *, Cuixia $\mathrm{Li}^{1}$ and Praveen Agarwal ${ }^{2,3}$ \\ 1 School of Mathematics, Yunnan Normal University, Kunming 650500, China; lixiatk@126.com \\ 2 Department of Mathrematics, Anand International College of Engineering, Jaipur 303012, India; \\ praveen.agarwal@anandice.ac.in \\ 3 Nonlinear Dynamics Research Center (NDRC), Ajman University, Ajman, United Arab Emirates \\ * Correspondence: slwuynnu@126.com \\ + This research was supported by National Natural Science Foundation of China (No.11961082).
}

check for

updates

Citation: Wu, S.; Li, C.; Agarwal, P. Relaxed Modulus-Based Matrix Splitting Methods for the Linear Complementarity Problem. Symmetry 2021, 13, 503. https://doi.org/ $10.3390 /$ sym 13030503

Academic Editor: Jan Awrejcewicz

Received: 12 February 2021

Accepted: 11 March 2021

Published: 19 March 2021

Publisher's Note: MDPI stays neutral with regard to jurisdictional claims in published maps and institutional affiliations.

\section{Copyright: (c) 2021 by the authors.} Licensee MDPI, Basel, Switzerland. This article is an open access article distributed under the terms and conditions of the Creative Commons Attribution (CC BY) license (https:// creativecommons.org/licenses/by/ $4.0 /)$.

\begin{abstract}
In this paper, we obtain a new equivalent fixed-point form of the linear complementarity problem by introducing a relaxed matrix and establish a class of relaxed modulus-based matrix splitting iteration methods for solving the linear complementarity problem. Some sufficient conditions for guaranteeing the convergence of relaxed modulus-based matrix splitting iteration methods are presented. Numerical examples are offered to show the efficacy of the proposed methods.
\end{abstract}

Keywords: linear complementarity problem; matrix splitting; iteration method; convergence

MSC: 90C33; 65F10; 65F50; 65G40

\section{Introduction}

In this paper, we focus on the iterative solution of the linear complementarity problem, abbreviated as ' $\operatorname{LCP}(q, A)^{\prime}$ ', whose form is

$$
w=A z+q \geq 0, z \geq 0 \text { and } z^{T} w=0,
$$

where $A \in \mathbb{R}^{n \times n}$ and $q \in \mathbb{R}^{n}$ are given, and $z \in \mathbb{R}^{n}$ is unknown, and for two $s \times t$ matrices $G=\left(g_{i j}\right)$ and $H=\left(h_{i j}\right)$ the order $G \geq(>) H$ means $g_{i j} \geq(>) h_{i j}$ for any $i$ and $j$. As is known, as a very useful tool in many fields, such as the free boundary problem, the contact problem, option pricing problem, nonnegative constrained least squares problems, see [1-5], $\operatorname{LCP}(q, A)$ is most striking in the articles, see [6-11].

Designing iteration methods to fast and economically computing the numerical solution of the $\operatorname{LCP}(q, A)$ is one of the hotspot nowadays, which were widely discussed in the articles, see $[1,2,8,9,12,13]$ for more details. Recently, by using $w=\frac{1}{\gamma} \Omega(|x|-x)$ and $z=\frac{1}{\gamma}(|x|+x)$ with $\gamma>0$ for the $\operatorname{LCP}(q, A)$, Bai in [14] expressed the $\operatorname{LCP}(q, A)$ as the fixed-point form

$$
(\Omega+M) x=N x+(\Omega-A)|x|-\gamma q,
$$

where $A=M-N$ is a splitting of matrix $A$ and $\Omega$ denotes a positive diagonal matrix, and then first desgined a class of modulus-based matrix splitting (MMS) iteration methods. Since the MMS method has the advantages of simple form and fast convergence rate, it was regarded as a powerful method of solving the $\operatorname{LCP}(q, A)$, and raises concerns. For several variants and applications of the MMS method, one can see [2,15-32].

In this paper, based on the MMS method, we will create a type of new iteration methods to solve the $\operatorname{LCP}(q, A)$. Our strategy is to introduce a relaxed matrix for both sides of Equation (2) and obtain a new equivalent fixed-point form of the $\operatorname{LCP}(q, A)$. Based on this new equivalent form, we can establish a class of relaxed modulus-based matrix splitting (RMMS) iteration methods for solving the $\operatorname{LCP}(q, A)$. This class of new iteration 
methods not alone inherits the virtues of the modulus-based methods. A more important one is that we can choose the relaxed matrix to enhance the computational efficiency of the classical MMS method in [14]. To guarantee the convergence of the RMMS iteration method, some sufficient conditions will be given under suitable conditions. Numerical examples are provided to verify that the RMMS iteration method are feasible and overmatch the classical MMS iteration method in terms of the computational efficiency.

The layout of this paper is as follows. In Section 2, for the sake of discussion in the rest of this paper, we provide some necessary definitions, lemmas and notations. In Section 3, we present a class of relaxed modulus-based matrix splitting (RMMS) iteration methods to solve the $\operatorname{LCP}(q, A)$. The convergence conditions of the RMMS iteration method are presented in Section 4. Numerical experiments with regard to the proposed methods compared to the classical MMS iteration method are reported in Section 5. In Section 6, we summarize this work. Finally, some simple discussions are given in Section 7.

\section{Preliminaries}

Some necessary definitions, lemmas and notations, which are used in the sequel discussions, are primitively introduced in this section.

Let $A=\left(a_{i j}\right) \in \mathbb{R}^{n \times n}$. Then it is named as a $Z$-matrix if $a_{i j} \leq 0(i \neq j)$; a nonsingular $M$-matrix if $A$ is a Z-matrix and $A^{-1} \geq 0$; its comparison matrix is $\langle A\rangle=\left(\langle a\rangle_{i j}\right)$ with $\langle a\rangle_{i j}$ being

$$
\langle a\rangle_{i j}=\left\{\begin{array}{c}
\left|a_{i j}\right| \text { for } i=j, \\
-\left|a_{i j}\right| \text { for } i \neq j,
\end{array} i, j=1,2, \ldots, n .\right.
$$

Further, a matrix $A=\left(a_{i j}\right) \in \mathbb{R}^{n \times n}$ is named as an $H$-matrix if $\langle A\rangle=\left(\langle a\rangle_{i j}\right)$ is an $M$-matrix; an $H_{+}$-matrix if $A$ is an $H$-matrix with $\operatorname{diag}(A)>0$; a $P$-matrix if all of its principal minors are positive $[33,34]$. In addition, we let $|A|=\left(\left|a_{i j}\right|\right)$.

Let $A=M-N$ be a splitting of matrix $A \in \mathbb{R}^{n \times n}$. Then it is named as an $M$-splitting if $M$ is a nonsingular $M$-matrix and $N \geq 0$; an $H$-splitting if $\langle M\rangle-|N|$ is a nonsingular $M$-matrix. As is known, if $A=M-N$ is an $M$-splitting and $A$ is a nonsingular $M$-matrix, then $\rho\left(M^{-1} N\right)<1$, where $\rho(\cdot)$ indicates the spectral radius (the maximum value of the absolute value of the eigenvalues) of the matrix, see [33,34]. Finally, $\|\cdot\|_{2}$ denotes the Euclidean norm on $\mathbb{R}^{n}$.

Lemma 1 ([19]). Let $A=\left(a_{i j}\right) \in \mathbb{R}^{n \times n}$ with $a_{i j} \geq 0$. If there exists $u \in \mathbb{R}^{n}$ with $u>0$ such that $A u<u$, then $\rho(A)<1$.

Lemma 2 ([35]). Let $A \in \mathbb{R}^{n \times n}$ be an $H$-matrix, $D$ be the diagonal part of the matrix $A$, and $A=D-B$. Then matrices $A$ and $|D|$ are nonsingular, $\left|A^{-1}\right| \leq\langle A\rangle^{-1}$ and $\rho\left(|D|^{-1}|B|\right)<1$.

Lemma 3 ([34]). Let $A$ be an M-matrix and $B$ be a Z-matrix with $A \leq B$. Then $B$ is an $M$-matrix.

In addition, there exists a famous result for the existence and uniqueness of the $\operatorname{LCP}(q, A)$, that is to say, the $\operatorname{LCP}(q, A)$ has a unique solution if and only if a matrix $A$ is a $P$-matrix, see [1]. Obviously, when $A$ is an $H_{+}$-matrix, the $\operatorname{LCP}(q, A)$ has a unique solution as well.

\section{Relaxed Modulus-Based Matrix Splitting Method}

In this section, we introduce a class of relaxed modulus-based matrix splitting (RMMS) iteration methods for solving the $\operatorname{LCP}(q, A)$. For this purpose, by introducing an identical equation $R x-R x=0$ for Equation (2), where $R \in \mathbb{R}^{n \times n}$ is a given relaxed matrix, then we obtain

$$
(\Omega+M) x=N x+(\Omega-A)|x|+R(x-x)-\gamma q,
$$

or,

$$
(\Omega+M-R) x=(N-R) x+(\Omega-A)|x|-\gamma q .
$$


Based on Equations (3) and (4), we can establish the following iteration method, which is named as a class of relaxed modulus-based matrix splitting (RMMS) iteration methods for the $\operatorname{LCP}(q, A)$.

Method 1. Let $A=M-N$ be a splitting of the matrix $A \in \mathbb{R}^{n \times n}$. Let $\gamma>0$ and matrix $\Omega+M-R$ be nonsingular, where $\Omega$ is a positive diagonal matrix and $R \in \mathbb{R}^{n \times n}$ is a given relaxed matrix. Given an initial vector $x^{(0)} \in \mathbb{R}^{n}$, compute $z^{(k+1)} \in \mathbb{R}^{n}$

$$
z^{(k+1)}=\frac{1}{\gamma}\left(\left|x^{(k+1)}\right|+x^{(k+1)}\right), k=0,1,2, \ldots,
$$

where $x^{(k+1)}$ can be obtained by solving the linear system

$$
(\Omega+M) x^{(k+1)}=N x^{(k)}+(\Omega-A)\left|x^{(k)}\right|+R\left(x^{(k+1)}-x^{(k)}\right)-\gamma q
$$

or

$$
(\Omega+M-R) x^{(k+1)}=(N-R) x^{(k)}+(\Omega-A)\left|x^{(k)}\right|-\gamma q .
$$

Clearly, when $R=0$, method 1 reduces to the well-known MMS iteration method in [14]. Similarly, by introducing an identical equation, $\mathrm{Wu}$ and $\mathrm{Li}$ in [22] presented twosweep modulus-based matrix splitting iteration methods for the $\operatorname{LCP}(q, A)$. The goal of introducing the relaxed matrix $R$ in (5) or (6) is that the computational efficiency of the RMMS iteration method may be better than the classical MMS iteration method in [14].

In fact, method 1 is a general framework of RMMS iteration methods for solving the $\operatorname{LCP}(q, A)$. This implies that we can construct some concrete forms of RMMS iteration methods by the specific splitting matrix of matrix $A$ and the iteration parameters. If we take

$$
M=\frac{1}{\alpha}(D-\beta L) \text { and } N=\frac{1}{\alpha}[(1-\alpha) D+(\alpha-\beta) L+\alpha U],
$$

where $D, L$ and $U$, respectively, are the diagonal, the strictly lower-triangular and the strictly upper-triangular parts of the matrix $A$, then this leads to the relaxed modulusbased AOR (RMAOR) iteration method

$$
(\Omega+D-\beta L-R) x^{(k+1)}=[(1-\alpha) D+(\alpha-\beta) L+\alpha U-R] x^{(k)}+(\Omega-\alpha A)\left|x^{(k)}\right|-\gamma \alpha q,
$$

with $z^{(k+1)}=\frac{1}{\gamma}\left(\left|x^{(k+1)}\right|+x^{(k+1)}\right)$. When $\alpha=\beta, \alpha=\beta=1$, and $\alpha=1, \beta=0$, respectively, the RMAOR method (7) can yield the corresponding relaxed modulus-based SOR (RMSOR) method, the relaxed modulus-based Gauss-Seidel (RMGS) method and the relaxed modulus-based Jacobi (RMJ) method.

\section{Convergence Analysis}

In this section, some sufficient conditions are given to guarantee the convergence of method 1 .

Theorem 1. Let $A=M-N$ be a splitting of the matrix $A \in \mathbb{R}^{n \times n}$ with $A$ being a P-matrix, and matrix $\Omega+M-R$ be nonsingular, where $\Omega$ is a positive diagonal matrix and $R \in \mathbb{R}^{n \times n}$ is a given relaxed matrix. Let

$$
\delta(R)=f(R)+g(R),
$$

where

$$
f(R)=\left\|(\Omega+M-R)^{-1}(N-R)\right\|_{2} \text { and } g(R)=\left\|(\Omega+M-R)^{-1}(\Omega-A)\right\|_{2} .
$$

When $\delta(R)<1$, Method 1 with $\gamma>0$ converges to the unique solution $z^{*} \in \mathbb{R}_{+}^{n}$ of the $\operatorname{LCP}(q, A)$ for an initial vector. 
Proof. Let $\left(z^{*}, w^{*}\right)$ be a solution pair of the $\operatorname{LCP}(q, A)$. Then $x^{*}=\frac{1}{2} \gamma\left(z^{*}-\Omega^{-1} w^{*}\right)$ satisfies

$$
(\Omega+M-R) x^{*}=(N-R) x^{*}+(\Omega-A)\left|x^{*}\right|-\gamma q .
$$

Based on (6) and (8), note that matrix $\Omega+M-R$ is nonsingular, we can obtain

$$
x^{(k+1)}-x^{*}=(\Omega+M-R)^{-1}\left((N-R)\left(x^{(k)}-x^{*}\right)+(\Omega-A)\left(\left|x^{(k)}\right|-\left|x^{*}\right|\right)\right) .
$$

This indicates that

$$
\begin{aligned}
\left\|x^{(k+1)}-x^{*}\right\|_{2}= & \left\|(\Omega+M-R)^{-1}\left((N-R)\left(x^{(k)}-x^{*}\right)+(\Omega-A)\left(\left|x^{(k)}\right|-\left|x^{*}\right|\right)\right)\right\|_{2} \\
\leq & \left\|(\Omega+M-R)^{-1}(N-R)\left(x^{(k)}-x^{*}\right)\right\|_{2} \\
& +\left\|(\Omega+M-R)^{-1}(\Omega-A)\left(\left|x^{(k)}\right|-\left|x^{*}\right|\right)\right\|_{2} \\
\leq & \left\|(\Omega+M-R)^{-1}(N-R)\right\|_{2}\left\|x^{(k)}-x^{*}\right\|_{2} \\
& +\left\|(\Omega+M-R)^{-1}(\Omega-A)\right\|_{2}\left\|x^{(k)}-x^{*}\right\|_{2} \\
= & (f(R)+g(R))\left\|x^{(k)}-x^{*}\right\|_{2} \\
= & \delta(R)\left\|x^{(k)}-x^{*}\right\|_{2} .
\end{aligned}
$$

Obviously, when $\delta(R)<1$, method 1 is convergent.

Since

$$
\begin{aligned}
& \left\|(\Omega+M-R)^{-1}(\Omega-A)\right\|_{2} \\
= & \left\|(\Omega+M-R)^{-1}(\Omega-M+N)\right\|_{2} \\
= & \left\|(\Omega+M-R)^{-1}(\Omega-M+N-R+R)\right\|_{2} \\
\leq & \left\|(\Omega+M-R)^{-1}(\Omega-M+R)\right\|_{2}+\left\|(\Omega+M-R)^{-1}(N-R)\right\|_{2},
\end{aligned}
$$

the following corollary can be obtained.

Corollary 1. Let $A=M-N$ be a splitting of the matrix $A \in \mathbb{R}^{n \times n}$ with A being a P-matrix, and matrix $\Omega+M-R$ be nonsingular, where $\Omega$ is a positive diagonal matrix and $R \in \mathbb{R}^{n \times n}$ is a given relaxed matrix. Let

$$
\bar{\delta}(R)=2 f(R)+\bar{g}(R),
$$

where

$$
f(R)=\left\|(\Omega+M-R)^{-1}(N-R)\right\|_{2} \text { and } \bar{g}(R)=\left\|(\Omega+M-R)^{-1}(\Omega-M+R)\right\|_{2} .
$$

When $\bar{\delta}(R)<1$, Method 1 with $\gamma>0$ converges to the unique solution $z^{*} \in \mathbb{R}_{+}^{n}$ of the $\operatorname{LCP}(q, A)$ for an initial vector.

Similar to the above proof, if we take $|\cdot|$ instead of $\|\cdot\|_{2}$, we can also obtain Corollary 2.

Corollary 2. Let $A=M-N$ be a splitting of the matrix $A \in \mathbb{R}^{n \times n}$ with $A$ being a P-matrix, and matrix $\Omega+M-R$ be nonsingular, where $\Omega$ is a positive diagonal matrix and $R \in \mathbb{R}^{n \times n}$ is a given relaxed matrix. Let

$$
\Phi=\left|(\Omega+M-R)^{-1}(N-R)\right|+\left|(\Omega+M-R)^{-1}(\Omega-A)\right|
$$

and

$$
\Psi=2\left|(\Omega+M-R)^{-1}(N-R)\right|+\left|(\Omega+M-R)^{-1}(\Omega-M+R)\right| .
$$

When $\rho(\Phi)<1$ or $\rho(\Psi)<1$, Method 1 with $\gamma>0$ converges to the unique solution $z^{*} \in \mathbb{R}_{+}^{n}$ of the $\operatorname{LCP}(q, A)$ for an initial vector. 
Theorem 2. Let $A=M-N$ be a splitting of the matrix $A \in \mathbb{R}^{n \times n}$ with $A$ being a P-matrix, and matrix $\Omega+M$ be nonsingular and matrix $I-\left|(\Omega+M)^{-1} R\right|$ be nonsingular $M$-matrix, where $\Omega$ is a positive diagonal matrix and $R \in \mathbb{R}^{n \times n}$ is a given relaxed matrix. Let

$$
\Theta=\left(I-\left|(\Omega+M)^{-1} R\right|\right)^{-1}\left(\left|(\Omega+M)^{-1} N\right|+\left|(\Omega+M)^{-1}(\Omega-A)\right|+\left|(\Omega+M)^{-1} R\right|\right) .
$$

When $\rho(\Theta)<1$, method 1 with $\gamma>0$ converges to the unique solution $z^{*} \in \mathbb{R}_{+}^{n}$ of the $\operatorname{LCP}(q, A)$ for an initial vector.

Proof. Based on (5) and (8), we have

$$
(\Omega+M)\left(x^{(k+1)}-x^{*}\right)=N\left(x^{(k)}-x^{*}\right)+(\Omega-A)\left(\left|x^{(k)}\right|-\left|x^{*}\right|\right)+R\left(\left(x^{(k+1)}-x^{*}\right)-\left(x^{(k)}-x^{*}\right)\right) .
$$

This implies

$$
\begin{aligned}
\left|x^{(k+1)}-x^{*}\right|= & \mid(\Omega+M)^{-1}\left(N\left(x^{(k)}-x^{*}\right)+(\Omega-A)\left(\left|x^{(k)}\right|-\left|x^{*}\right|\right)\right. \\
& \left.+R\left(\left(x^{(k+1)}-x^{*}\right)-\left(x^{(k)}-x^{*}\right)\right)\right) \mid \\
= & \mid(\Omega+M)^{-1} N\left(x^{(k)}-x^{*}\right)+(\Omega+M)^{-1}(\Omega-A)\left(\left|x^{(k)}\right|-\left|x^{*}\right|\right) \\
& \left.+(\Omega+M)^{-1} R\left(\left(x^{(k+1)}-x^{*}\right)-\left(x^{(k)}-x^{*}\right)\right)\right) \mid \\
\leq & \left|(\Omega+M)^{-1} N\right| \cdot\left|x^{(k)}-x^{*}\right|+\left|(\Omega+M)^{-1}(\Omega-A)\right| \cdot|| x^{(k)}|-| x^{*}|| \\
& +\left|(\Omega+M)^{-1} R\right| \cdot\left|x^{(k+1)}-x^{*}\right|+\left|(\Omega+M)^{-1} R\right| \cdot\left|x^{(k)}-x^{*}\right| \\
\leq & \left|(\Omega+M)^{-1} N\right| \cdot\left|x^{(k)}-x^{*}\right|+\left|(\Omega+M)^{-1}(\Omega-A)\right| \cdot\left|x^{(k)}-x^{*}\right| \\
& +\left|(\Omega+M)^{-1} R\right| \cdot\left|x^{(k+1)}-x^{*}\right|+\left|(\Omega+M)^{-1} R\right| \cdot\left|x^{(k)}-x^{*}\right| .
\end{aligned}
$$

Further, we have

$$
\left|x^{(k+1)}-x^{*}\right| \leq \Theta(R)\left(\left|x^{(k)}-x^{*}\right|\right)
$$

where

$$
\Theta=\left(I-\left|(\Omega+M)^{-1} R\right|\right)^{-1}\left(\left|(\Omega+M)^{-1} N\right|+\left|(\Omega+M)^{-1}(\Omega-A)\right|+\left|(\Omega+M)^{-1} R\right|\right) .
$$

Clearly, when $\rho(\Theta)<1$, Method 1 is convergent.

Similarly, we have Corollary 3.

Corollary 3. Let $A=M-N$ be a splitting of the matrix $A \in \mathbb{R}^{n \times n}$ with $A$ being a P-matrix, and matrix $\Omega+M$ be nonsingular and $I-\left|(\Omega+M)^{-1} R\right|$ be nonsingular $M$-matrix, where $\Omega$ is a positive diagonal matrix and $R \in \mathbb{R}^{n \times n}$ is a given relaxed matrix. Let

$$
\bar{\Theta}=\left(I-\left|(\Omega+M)^{-1} R\right|\right)^{-1}\left(2\left|(\Omega+M)^{-1} N\right|+\left|(\Omega+M)^{-1}(\Omega-M)\right|+\left|(\Omega+M)^{-1} R\right|\right) .
$$

When $\rho(\bar{\Theta})<1$, method 1 with $\gamma>0$ converges to the unique solution $z^{*} \in \mathbb{R}_{+}^{n}$ of the $\operatorname{LCP}(q, A)$ for an initial vector.

Theorem 3. Let $A=M-N$ be a splitting of the matrix $A=\left(a_{i j}\right) \in \mathbb{R}^{n \times n}$, where $A$ is an $H_{+}$-matrix, and $\langle M-R\rangle-|N-R|$ be an M-matrix with $R=\left(r_{i j}\right) \in \mathbb{R}^{n \times n}$. Let matrix $\Omega=$ $\left(\omega_{i j}\right) \in \mathbb{R}^{n \times n}$ satisfy $\omega_{i i} \geq a_{i i}$ and $0 \leq \omega_{i j} \leq \frac{1}{2}\left(\left|m_{i j}-r_{i j}\right|+\left|n_{i j}-r_{i j}\right|-\left|a_{i j}\right|\right)$. Then method 1 with $\gamma>0$ converges to the unique solution $z^{*} \in \mathbb{R}_{+}^{n}$ of the $\operatorname{LCP}(q, A)$ for an initial vector.

Proof. First, we prove that $\langle\Omega+M-R\rangle$ is an $M$-matrix. In fact, since

$$
0 \leq \omega_{i j} \leq \frac{1}{2}\left(\left|m_{i j}-r_{i j}\right|+\left|n_{i j}-r_{i j}\right|-\left|a_{i j}\right|\right),
$$


we obtain

$$
\begin{aligned}
2\left|m_{i j}-r_{i j}\right|+\left|n_{i j}-r_{i j}\right| & \geq \omega_{i j}+\left|a_{i j}\right|+\omega_{i j}+\left|m_{i j}-r_{i j}\right| \\
& \geq\left|\omega_{i j}-a_{i j}\right|+\left|\omega_{i j}+m_{i j}-r_{i j}\right| .
\end{aligned}
$$

Further, we have

$$
2\left|m_{i j}-r_{i j}\right|+\left|n_{i j}-r_{i j}\right|-\left|\omega_{i j}-a_{i j}\right| \leq\left|\omega_{i j}+m_{i j}-r_{i j}\right|,
$$

which is equal to

$$
-2\left|m_{i j}-r_{i j}\right|-\left|n_{i j}-r_{i j}\right|+\left|\omega_{i j}-a_{i j}\right| \leq-\left|\omega_{i j}+m_{i j}-r_{i j}\right| .
$$

In addition,

$$
\omega_{i i}+m_{i i}-r_{i i}=\omega_{i i}-\left|m_{i i}-r_{i i}\right|+\left|n_{i i}-r_{i i}\right|+2\left|m_{i i}-r_{i i}\right|-\left|n_{i i}-r_{i i}\right|>0 .
$$

Therefore,

$$
\langle\Omega+M-R\rangle \geq \Delta+2\langle M+R\rangle-|N+R|,
$$

where matrix $\Delta=\left(\delta_{i j}\right) \in \mathbb{R}^{n \times n}$ satisfy

$$
\left(\delta_{i j}\right)=\left\{\begin{array}{l}
\omega_{i i}-\left|m_{i i}-r_{i i}\right|+\left|n_{i i}-r_{i i}\right| \geq 0 \text { for } i=j, \\
\left|\omega_{i j}-a_{i j}\right| \text { for } i \neq j,
\end{array} i, j=1,2, \ldots, n .\right.
$$

It noted that

$$
\Delta+2\langle M-R\rangle-|N-R| \geq 2(\langle M-R\rangle-|N-R|) .
$$

Based on Lemma 3, matrix $\Delta+2\langle M-R\rangle-|N-R|$ is an M-matrix. Based on (10) and (11), we have

$$
2(\langle M-R\rangle-|N-R|) \leq\langle\Omega+M-R\rangle,
$$

which implies that $\langle\Omega+M-R\rangle$ is an $H_{+}$matrix.

Based on (9) and Lemma 2, we have

$$
\begin{aligned}
\left|x^{(k+1)}-x^{*}\right| & =\left|(\Omega+M-R)^{-1}\left((N-R)\left(x^{(k)}-x^{*}\right)+(\Omega-A)\left(\left|x^{(k)}\right|-\left|x^{*}\right|\right)\right)\right| \\
& \leq\left|(\Omega+M-R)^{-1}\right| \cdot\left|(N-R)\left(x^{(k)}-x^{*}\right)+(\Omega-A)\left(\left|x^{(k)}\right|-\left|x^{*}\right|\right)\right| \\
& \leq\left|(\Omega+M-R)^{-1}\right|(|N-R|+|\Omega-A|)\left|x^{(k)}-x^{*}\right| \\
& \leq\langle\Omega+M-R\rangle^{-1}(|N-R|+|\Omega-A|)\left|x^{(k)}-x^{*}\right| \\
& =\langle\Omega+M-R\rangle^{-1}(\langle\Omega+M-R\rangle-\langle\Omega+M-R\rangle+|N-R|+|\Omega-A|)\left|x^{(k)}-x^{*}\right| \\
& =\left[I-\langle\Omega+M-R\rangle^{-1}(\langle\Omega+M-R\rangle-|N-R|-|\Omega-A|)\right]\left|x^{(k)}-x^{*}\right| \\
& \leq\left[I-\langle\Omega+M-R\rangle^{-1}(2\langle M-R\rangle-2|N-R|+\Delta-|\Omega-A|)\right]\left|x^{(k)}-x^{*}\right| .
\end{aligned}
$$

By calculation, it is easy to obtain that matrix $\Delta-|\Omega-A|$ is a nonnegative diagonal matrix. It follows that matrix

$$
2\langle M-R\rangle-2|N-R|+\Delta-|\Omega-A|
$$

is an $M$-matrix. Further, there exists a positive vector $u$ such that

$$
(2\langle M-R\rangle-2|N-R|+\Delta-|\Omega-A|) u>0 .
$$


Therefore,

$$
\langle\Omega+M-R\rangle^{-1}(2\langle M-R\rangle-2|N-R|+\Delta-|\Omega-A|) u>0 .
$$

Let

$$
W=I-\langle\Omega+M-R\rangle^{-1}(2\langle M-R\rangle-2|N-R|+\Delta-|\Omega-A|) .
$$

Then

$$
W u=\left[I-\langle\Omega+M-R\rangle^{-1}(2\langle M-R\rangle-2|N-R|+\Delta-|\Omega-A|)\right] u<u .
$$

Based on Lemma 1, we can obtain that $\rho(W)<1$. which completes the proof.

When matrix $R$ is a diagonal matrix, Corollary 4 can be obtained.

Corollary 4. Let $A=M-N$ be a splitting of the matrix $A=\left(a_{i j}\right) \in \mathbb{R}^{n \times n}$, where $A$ is an $H_{+}$-matrix, and $\langle M-R\rangle-|N-R|$ be an M-matrix with $R=\left(r_{i j}\right) \in \mathbb{R}^{n \times n}$ being a diagonal matrix. Let matrix $\Omega=\left(\omega_{i j}\right) \in \mathbb{R}^{n \times n}$ satisfy $\omega_{i i} \geq a_{i i}$ and $0 \leq \omega_{i j} \leq \frac{1}{2}\left(\left|m_{i j}\right|+\left|n_{i j}\right|-\left|a_{i j}\right|\right)$. Then Method 1 with $\gamma>0$ converges to the unique solution $z^{*} \in \mathbb{R}_{+}^{n}$ of the $\operatorname{LCP}(q, A)$ for an initial vector.

When matrix $R$ is a zero matrix, Corollary 4 reduces to the following result, which is a main result in [36].

Corollary 5. [36] Let $A=M-N$ be a splitting of the matrix $A=\left(a_{i j}\right) \in \mathbb{R}^{n \times n}$, where $A$ is an $H_{+}$-matrix, and $\langle M\rangle-|N|$ be an M-matrix. Let $\Omega=\left(\omega_{i j}\right) \in \mathbb{R}^{n \times n}$ satisfy $\omega_{i i} \geq a_{i i}$ and $0 \leq \omega_{i j} \leq \frac{1}{2}\left(\left|m_{i j}\right|+\left|n_{i j}\right|-\left|a_{i j}\right|\right)$. Then method 1 with $R=0$ and $\gamma>0$ converges to the unique solution $z^{*} \in \mathbb{R}_{+}^{n}$ of the $\operatorname{LCP}(q, A)$ for an initial vector.

Further, when matrix $R$ is a zero matrix and $\Omega=\left(\omega_{i j}\right) \in \mathbb{R}^{n \times n}$ is a positive diagonal matrix, Corollary 4 reduces to the following result, which is a main result in [37].

Corollary 6. [37] Let $A=M-N$ be a splitting of the matrix $A=\left(a_{i j}\right) \in \mathbb{R}^{n \times n}$, where $A$ is an $H_{+}$-matrix, and $\langle M\rangle-|N|$ be an M-matrix. Let the positive diagonal matrix $\Omega=\left(\omega_{i j}\right) \in \mathbb{R}^{n \times n}$ satisfy $\omega_{i i} \geq a_{i i}$. Then Method 1 with $R=0$ and $\gamma>0$ converges to the unique solution $z^{*} \in \mathbb{R}_{+}^{n}$ of the $\operatorname{LCP}(q, A)$ for an initial vector.

Theorem 4. Let $A=D-L-U=D-B$ and $\langle A\rangle=D-|L|-|U|$, where $A \in \mathbb{R}^{n \times n}$ is an $H_{+}$-matrix. Assume that the positive diagonal matrix $\Omega$ satisfies $\Omega \geq D$, matrix $R$ is a lowertriangular matrix with $\operatorname{diag}(R) \leq 0$, and $\rho:=\rho\left(D^{-1}|B|+\left|L_{R}\right|\right)<1$, where $L_{R}=R-\operatorname{diag}(R)$. Then for an initial vector, the RMAOR iteration method with $\gamma>0$ is convergent if the parameters $\alpha$ and $\beta$ satisfy

$$
0 \leq \max \{\alpha, \beta\} \rho<\min \{1, \alpha\}
$$

Proof. From the proof of Theorem 3, we take

$$
M=\frac{1}{\alpha}(D-\beta L) \text { and } N=\frac{1}{\alpha}[(1-\alpha) D+(\alpha-\beta) L+\alpha U] .
$$

Since $\Omega \geq D>0$ and $\operatorname{diag}(R) \leq 0$, obviously, matrix $\Omega-R+\frac{1}{\alpha}(D-\beta L)$ is an $H_{+}$-matrix. Based on Lemma 2, we have

$$
\left|\left(\Omega-R+\frac{1}{\alpha}(D-\beta L)\right)^{-1}\right| \leq\left\langle\Omega-R+\frac{1}{\alpha}(D-\beta L)\right\rangle^{-1}=\left(\Omega-D_{R}+D-\left|L_{R}+\frac{\alpha}{\beta} L\right|\right)^{-1}
$$


with $D_{R}=\operatorname{diag}(R)$ and $L_{R}=R-\operatorname{diag}(R)$. Let

$$
\hat{W}=|\Omega-R+M|^{-1}(|N|+|R|+|\Omega-A|) .
$$

Then by the simple computations we have

$$
\begin{aligned}
\hat{W}= & \left|\Omega-R+\frac{1}{\alpha}(D-\beta L)\right|^{-1}\left(\left|\frac{1}{\alpha}[(1-\alpha) D+(\alpha-\beta) L+\alpha U]\right|+|R|+|\Omega-A|\right) \\
= & |\alpha \Omega-\alpha R+D-\beta L|^{-1}(|(1-\alpha) D+(\alpha-\beta) L+\alpha U|+\alpha|R|+\alpha|\Omega-A|) \\
= & \left|\alpha \Omega-\alpha D_{R}-\alpha L_{R}+D-\beta L\right|^{-1}(|(1-\alpha) D+(\alpha-\beta) L+\alpha U|+\alpha|R|+\alpha|\Omega-A|) \\
\leq & \left\langle\alpha \Omega-\alpha D_{R}+D-\alpha L_{R}-\beta L\right\rangle^{-1}(|(1-\alpha) D+(\alpha-\beta) L+\alpha U|+\alpha|R|+\alpha|\Omega-A|) \\
= & \left(\alpha \Omega-\alpha D_{R}+D-\left|\alpha L_{R}+\beta L\right|\right)^{-1}(|(1-\alpha) D+(\alpha-\beta) L+\alpha U|+\alpha|R|+\alpha|\Omega-A|) \\
\leq & \left(\alpha \Omega-\alpha D_{R}+D-\left|\alpha L_{R}\right|-|\beta L|\right)^{-1}(|(1-\alpha) D+(\alpha-\beta) L+\alpha U|+\alpha|R|+\alpha|\Omega-A|) \\
= & \left(\alpha \Omega-\alpha D_{R}+D-\left|\alpha L_{R}\right|-|\beta L|\right)^{-1}\left(\alpha \Omega-\alpha D_{R}+D-\left|\alpha L_{R}\right|-|\beta L|\right. \\
& \left.-\left(\alpha \Omega-\alpha D_{R}+D-\left|\alpha L_{R}\right|-|\beta L|\right)+|(1-\alpha) D+(\alpha-\beta) L+\alpha U|+\alpha|R|+\alpha|\Omega-A|\right) \\
= & I-\left(\alpha \Omega-\alpha D_{R}+D-\alpha\left|L_{R}\right|-\beta|L|\right)^{-1}\left(\alpha \Omega-\alpha D_{R}+D-\left|\alpha L_{R}\right|-|\beta L|\right. \\
& -|(1-\alpha) D+(\alpha-\beta) L+\alpha U|-\alpha|R|-\alpha|\Omega-A|) \\
= & I-\left(\alpha \Omega-\alpha D_{R}+D-\alpha\left|L_{R}\right|-\beta|L|\right)^{-1}\left(\alpha \Omega-\alpha D_{R}+D-\left|\alpha L_{R}\right|-|\beta L|\right. \\
& \left.-|1-\alpha| D-|\alpha B-\beta L|-\alpha\left|D_{R}\right|-\alpha\left|L_{R}\right|-\alpha(\Omega-D)-\alpha|B|\right) \\
= & I-\left(\alpha \Omega-\alpha D_{R}+D-\alpha\left|L_{R}\right|-\beta|L|\right)^{-1}((1+\alpha)-|1-\alpha|) D-2 \alpha\left|L_{R}\right| \\
& -|\alpha B-\beta L|-\beta|L|-\alpha|B|)
\end{aligned}
$$

Since

$$
(1+\alpha-|1-\alpha|)=2 \min \{1, \alpha\}
$$

and

$$
\begin{aligned}
|\alpha B-\beta L|+\alpha|B|+\beta|L| & =|\alpha L+\alpha U-\beta L|+\alpha|U|+\alpha|L|+\beta|L| \\
& =(|\alpha-\beta|+\alpha+\beta)|L|+2 \alpha|U| \\
& \leq 2 \max \{\alpha, \beta\}|B|,
\end{aligned}
$$

then

$$
\hat{W} \leq \tilde{W},
$$

where

$\tilde{W}=I-2\left(\alpha \Omega-\alpha D_{R}+D-\alpha\left|L_{R}\right|-\beta|L|\right)^{-1} D\left(\min \{1, \alpha\} I-\max \{\alpha, \beta\} D^{-1}\left(|B|+\left|L_{R}\right|\right)\right)$.

Note that $\rho\left(D^{-1}\left(|B|+\left|L_{R}\right|\right)\right)<1$. Then there exists an arbitrary small number $\varepsilon>0$ such that

$$
\rho_{\varepsilon}:=\rho\left(J_{\varepsilon}\right)<1,
$$

where $J_{\varepsilon}:=D^{-1}\left(|B|+\left|L_{R}\right|\right)+\epsilon e e^{T}$ and $e:=(1, \ldots, 1)^{T} \in \mathbb{R}^{n}$. Based on Perron-Frobenius theorem in [34], there exists a positive vector $u_{\varepsilon} \in \mathbb{R}^{n}$ such that

$$
J_{\mathcal{\varepsilon}} u_{\mathcal{\varepsilon}}=\rho_{\mathcal{\varepsilon}} u_{\mathcal{\varepsilon}} .
$$

Therefore,

$$
\begin{aligned}
\tilde{W} u_{\varepsilon} & \leq u_{\varepsilon}-2\left(\alpha \Omega-\alpha D_{R}+D-\alpha\left|L_{R}\right|-\beta|L|\right)^{-1} D\left(\min \{1, \alpha\} u_{\varepsilon}-\max \{\alpha, \beta\} J_{\mathcal{\varepsilon}} u_{\varepsilon}\right) \\
& =u_{\varepsilon}-2\left(\min \{1, \alpha\}-\max \{\alpha, \beta\} \rho_{\varepsilon}\right)\left(\alpha \Omega-\alpha D_{R}+D-\alpha\left|L_{R}\right|-\beta|L|\right)^{-1} D u_{\varepsilon} \\
& <v_{\varepsilon} .
\end{aligned}
$$


Based on Lemma 1, we can obtain that $\rho(\hat{W}) \leq \rho(\tilde{W})<1$, which implies that the result of Theorem 4 is true.

When matrix $R$ in Theorem 4 is a nonpositive diagonal matrix, Theorem 4 reduces to the following result.

Corollary 7. Let $A=D-L-U=D-B$ and $\langle A\rangle=D-|L|-|U|$, where $A \in \mathbb{R}^{n \times n}$ is an $H_{+}$-matrix. Assume that the positive diagonal matrix $\Omega$ satisfies $\Omega \geq D$, matrix $R$ is a nonpositive diagonal matrix and $\rho:=\rho\left(D^{-1}|B|\right)$. Then for an initial vector, the RMAOR iteration method with $\gamma>0$ is convergent if the parameters $\alpha$ and $\beta$ satisfy

$$
0 \leq \max \{\alpha, \beta\} \rho<\min \{1, \alpha\} .
$$

\section{Numerical Experiments}

In this section, we utilize two examples to illustrate the computational efficiency of the RMMS iteration method in terms of iteration steps (IT) and elapsed CPU time (CPU) in seconds, and the following norm of absolute residual vectors (RES)

$$
\operatorname{RES}\left(z^{(k)}\right):=\left\|\min \left(A z^{(k)}+q, z^{(k)}\right)\right\|_{2}
$$

where the minimum is componentwisely taken.

To show the advantages of the RMMS iteration method, we compare the RMMS iteration method with the classical MMS iteration method. During these tests, all initial vectors are chosen to be

$$
x^{(0)}=(1,0,1,0, \ldots, 1,0, \ldots)^{T} \in \mathbb{R}^{n},
$$

all the iterations are stopped once $\operatorname{RES}\left(z^{(k)}\right) \leq 10^{-5}$ or the number of iteration exceeds 500 . For convenience, here we consider the relaxed modulus-based SOR (RMSOR) method and the modulus-based SOR (MSOR) method. The basis of this comparison is that the modulusbased SOR (MSOR) method in [14] outperforms other forms of the modulus-based matrix splitting iteration method, the projected relaxation methods and the modified modulus method. In actual implementations, we take $\Omega=D$ and $\gamma=2$ for the RMSOR method and the MSOR method. All of computations are performed in MATLAB 7.0. In addition, in the following tables, ' - ' denotes that the iteration steps exceed 500 or the residual norms exceed $10^{-5}$.

In our computations, we take the following two examples, which were considered in $[14,18]$. Parts of two examples are symmetry, see case 1 of Example 1 and the diagonal matrix of Example 2. Of course, we consider the non-symmetry case, see case 2 of Example 1 and Example 2.

Example 1 ([14]). Let the $\operatorname{LCP}(q, A)$ be given by $q=-A z^{*}$ and $A=\hat{A}+\mu I$, where

$$
\hat{A}=\operatorname{tridiag}(-r I, S,-t I)=\left[\begin{array}{cccccc}
S & -t I & 0 & \cdots & 0 & 0 \\
-r I & S & -t I & \cdots & 0 & 0 \\
0 & -r I & S & \cdots & 0 & 0 \\
\vdots & \vdots & \vdots & \ddots & \vdots & \vdots \\
0 & 0 & 0 & \cdots & S & -t I \\
0 & 0 & 0 & \cdots & -r I & S
\end{array}\right] \in \mathbb{R}^{n \times n}
$$


with

$$
S=\operatorname{tridiag}(-r, 4,-t)=\left[\begin{array}{cccccc}
4 & -t & 0 & \cdots & 0 & 0 \\
-r & 4 & -t & \cdots & 0 & 0 \\
0 & -r & 4 & \cdots & 0 & 0 \\
\vdots & \vdots & \vdots & \ddots & \vdots & \vdots \\
0 & 0 & 0 & \cdots & 4 & -t \\
0 & 0 & 0 & \cdots & -r & 4
\end{array}\right] \in \mathbb{R}^{m \times m}
$$

and

$$
z^{*}=(1,2,1,2, \ldots, 1,2, \ldots)^{T} \in \mathbb{R}^{n}
$$

is the unique solution of the $\operatorname{LCP}(q, A)$.

Example 2 (([18]). Let the $\operatorname{LCP}(q, A)$ be given by

$$
A=\left[\begin{array}{ccccccc}
W & -I & -I & 0 & \cdots & 0 & 0 \\
0 & W & -I & -I & \cdots & 0 & 0 \\
0 & 0 & W & -I & \cdots & 0 & 0 \\
\vdots & \vdots & \vdots & \vdots & \ddots & \vdots & \vdots \\
0 & 0 & 0 & 0 & \cdots & W & -I \\
0 & 0 & 0 & 0 & \cdots & 0 & W
\end{array}\right] \in \mathbb{R}^{n \times n} \text { and } q=\left[\begin{array}{c}
-1 \\
1 \\
-1 \\
\vdots \\
(-1)^{n-1} \\
(-1)^{n}
\end{array}\right] \in \mathbb{R}^{n},
$$

with

$$
W=\operatorname{tridiag}(-1,4,-1)=\left[\begin{array}{cccccc}
4 & -1 & 0 & \cdots & 0 & 0 \\
-1 & 4 & -1 & \cdots & 0 & 0 \\
0 & -1 & 4 & \cdots & 0 & 0 \\
\vdots & \vdots & \vdots & \ddots & \vdots & \vdots \\
0 & 0 & 0 & \cdots & 4 & -1 \\
0 & 0 & 0 & \cdots & -1 & 4
\end{array}\right] \in \mathbb{R}^{m \times m}
$$

In Examples 1 and 2, the value of $m$ is chosen to be a prescribed positive integer, and then $n=m^{2}$. For Example 1, we consider two cases: one is the symmetric case and the other is the nonsymmetric case. For the former, we take $r=t=1$; for the latter, we take $r=1.5$ and $t=0.5$. In the implementations, the value of the iteration parameter $\alpha$ used in both the RMSOR method and the MSOR method is chosen to be 1.3. For convenience, the value of matrix is chosen to be $R=2 I$ for the RMSOR method, where I is the identity matrix.

For Example 1, for different problem sizes of $m$ and the different values of $\mu$, the numerical results (including IT, CPU and RES) for the RMSOR method and the MSOR method are listed in Table 1 when $r=t=1$. Clearly, the RMSOR method and the MSOR method can rapidly compute a satisfactory approximation to the solution of the $\operatorname{LCP}(q, A)$.

From the numerical results in Table 1, fixed the value of $\mu$, the iteration steps and the CPU times of the RMSOR method and the MSOR method are incremental when the problem size $n=m^{2}$ is increasing. Whereas, fixing the value of the problem size $n=m^{2}$, the iteration steps and the CPU times of the RMSOR method and the MSOR method are descended when the value of $\mu$ is increasing. This implies that both may be fit for the larger $\mu$ when as a solver for solving the $\operatorname{LCP}(q, A)$.

Base on the presented numerical results in Table 1, our numerical experiment show that the RMSOR method compared to the MSOR method requires less iteration steps and CPU times. This shows that when both RMSOR and MSOR methods are used to solve the $\operatorname{LCP}(q, A)$, the former is superior the latter.

Table 2 presents the numerical results of the nonsymmetric case of Example 1. Specifically, some numerical results (including IT, CPU and RES) for the RMSOR method and the MSOR method for different problem sizes of $m$ and the different values of $\mu$ are listed when $r=1.5$ and $t=0.5$. Table 2 shows that the RMSOR method and the MSOR method can still rapidly compute a satisfactory approximation to the solution of the $\operatorname{LCP}(q, A)$. 
Table 1. Numerical results for Example 1 with $r=t=1$.

\begin{tabular}{|c|c|c|c|c|c|c|c|}
\hline & & $m$ & 20 & 30 & 40 & 50 & 60 \\
\hline \multirow{6}{*}{$\mu=2$} & \multirow{3}{*}{ RMSOR } & IT & 25 & 25 & 26 & 27 & 27 \\
\hline & & CPU & 0.0313 & 0.0313 & 0.0625 & 0.0625 & 0.1094 \\
\hline & & RES & $5.908 \times 10^{-6}$ & $5.819 \times 10^{-6}$ & $8.472 \times 10^{-6}$ & $6.027 \times 10^{-6}$ & $7.469 \times 10^{-6}$ \\
\hline & \multirow{3}{*}{ MSOR } & IT & 51 & 53 & 53 & 54 & 55 \\
\hline & & CPU & 0.0313 & 0.0781 & 0.0938 & 0.1094 & 0.1875 \\
\hline & & RES & $8.427 \times 10^{-6}$ & $7.753 \times 10^{-6}$ & $9.798 \times 10^{-6}$ & $8.895 \times 10^{-6}$ & $7.782 \times 10^{-6}$ \\
\hline \multirow{6}{*}{$\mu=4$} & \multirow{3}{*}{ RMSOR } & IT & 20 & 20 & 21 & 21 & 21 \\
\hline & & CPU & 0.0313 & 0.0313 & 0.0625 & 0.0625 & 0.0781 \\
\hline & & RES & $7.566 \times 10^{-6}$ & $9.882 \times 10^{-6}$ & $5.930 \times 10^{-6}$ & $6.748 \times 10^{-6}$ & $7.476 \times 10^{-6}$ \\
\hline & \multirow{3}{*}{ MSOR } & IT & 34 & 35 & 35 & 35 & 36 \\
\hline & & CPU & 0.0313 & 0.0625 & 0.0781 & 0.1094 & 0.0938 \\
\hline & & RES & $7.574 \times 10^{-6}$ & $6.854 \times 10^{-6}$ & $8.276 \times 10^{-6}$ & $9.488 \times 10^{-6}$ & $7.083 \times 10^{-6}$ \\
\hline \multirow{6}{*}{$\mu=8$} & \multirow{3}{*}{ RMSOR } & IT & 18 & 18 & 18 & 18 & 18 \\
\hline & & $\mathrm{CPU}$ & 0.0156 & 0.0313 & 0.0313 & 0.0469 & 0.0625 \\
\hline & & RES & $4.675 \times 10^{-6}$ & $5.998 \times 10^{-6}$ & $7.078 \times 10^{-6}$ & $8.014 \times 10^{-6}$ & $8.851 \times 10^{-6}$ \\
\hline & \multirow{3}{*}{ MSOR } & IT & 24 & 24 & 25 & 25 & 25 \\
\hline & & $\mathrm{CPU}$ & 0.0313 & 0.0313 & 0.0625 & 0.0625 & 0.0781 \\
\hline & & RES & $7.476 \times 10^{-6}$ & $9.658 \times 10^{-6}$ & $6.343 \times 10^{-6}$ & $7.197 \times 10^{-6}$ & $7.961 \times 10^{-6}$ \\
\hline
\end{tabular}

Table 2. Numerical results for Example 1 with $r=1.5$ and $t=0.5$.

\begin{tabular}{|c|c|c|c|c|c|c|c|}
\hline & & $m$ & 20 & 30 & 40 & 50 & 60 \\
\hline \multirow{6}{*}{$\mu=2$} & \multirow{3}{*}{ RMSOR } & IT & 21 & 21 & 22 & 22 & 23 \\
\hline & & CPU & 0.0156 & 0.0313 & 0.0469 & 0.0469 & 0.0781 \\
\hline & & RES & $4.669 \times 10^{-6}$ & $9.734 \times 10^{-6}$ & $7.082 \times 10^{-6}$ & $9.528 \times 10^{-6}$ & $5.772 \times 10^{-6}$ \\
\hline & \multirow{3}{*}{ MSOR } & IT & 28 & 28 & 29 & 29 & 29 \\
\hline & & CPU & 0.0313 & 0.0313 & 0.0625 & 0.0938 & 0.1094 \\
\hline & & RES & $6.588 \times 10^{-6}$ & $8.501 \times 10^{-6}$ & $6.252 \times 10^{-6}$ & $7.091 \times 10^{-6}$ & $7.840 \times 10^{-6}$ \\
\hline \multirow{6}{*}{$\mu=4$} & \multirow{3}{*}{ RMSOR } & IT & 15 & 15 & 16 & 16 & 16 \\
\hline & & CPU & 0.0156 & 0.0156 & 0.0469 & 0.0625 & 0.0625 \\
\hline & & RES & $8.430 \times 10^{-6}$ & $4.328 \times 10^{-6}$ & $5.092 \times 10^{-6}$ & $5.756 \times 10^{-6}$ & $6.351 \times 10^{-6}$ \\
\hline & \multirow{3}{*}{ MSOR } & IT & 23 & 23 & 24 & 24 & 24 \\
\hline & & $\mathrm{CPU}$ & 0.0313 & 0.0469 & 0.0625 & 0.0781 & 0.0781 \\
\hline & & RES & $6.771 \times 10^{-6}$ & $8.646 \times 10^{-6}$ & $5.609 \times 10^{-6}$ & $6.345 \times 10^{-6}$ & $7.004 \times 10^{-6}$ \\
\hline \multirow{6}{*}{$\mu=8$} & \multirow{3}{*}{ RMSOR } & IT & 15 & 15 & 15 & 15 & 15 \\
\hline & & $\mathrm{CPU}$ & 0.0156 & 0.0313 & 0.0313 & 0.0313 & 0.0469 \\
\hline & & RES & $4.011 \times 10^{-6}$ & $5.077 \times 10^{-6}$ & $5.955 \times 10^{-6}$ & $6.719 \times 10^{-6}$ & $7.405 \times 10^{-6}$ \\
\hline & \multirow{3}{*}{ MSOR } & IT & 19 & 19 & 20 & 20 & 20 \\
\hline & & $\mathrm{CPU}$ & 0.0156 & 0.0313 & 0.0625 & 0.0625 & 0.0938 \\
\hline & & RES & $7.112 \times 10^{-6}$ & $8.999 \times 10^{-6}$ & $4.977 \times 10^{-6}$ & $5.617 \times 10^{-6}$ & $6.191 \times 10^{-6}$ \\
\hline
\end{tabular}


From Table 2, these numerical results further verify the observed results from Table 1. Our numerical experiments show that the computational efficiency of the RMSOR method is better than the MSOR method. It is noted that compared with the symmetric case, the iteration steps and the CPU times of the RMSOR method and the MSOR method slightly decrease in the nonsymmetric case.

Table 3 presents the numerical results of Example 2. To compare the RMSOR method with the MSOR method, Table 3 lists the numerical results (including IT, CPU and RES) for the different values of $\mu$ under the same iteration parameter $\alpha$. From the presented numerical results in Table 3, our numerical experiments show that that the RMSOR method requires less iteration steps and CPU times than the MSOR method. The numerical results in Table 3 confirm that the RMSOR method is still superior to the MSOR method.

Table 3. Numerical results for Example 2.

\begin{tabular}{|c|c|c|c|c|c|c|}
\hline & & $\alpha$ & 1.4 & 1.6 & 1.8 & 2 \\
\hline \multirow{6}{*}{$m=20$} & \multirow{3}{*}{ RMSOR } & IT & 33 & 30 & 43 & 80 \\
\hline & & CPU & 0.0313 & 0.0313 & 0.0469 & 0.0938 \\
\hline & & RES & $7.452 \times 10^{-6}$ & $9.803 \times 10^{-6}$ & $9.105 \times 10^{-6}$ & $9.712 \times 10^{-6}$ \\
\hline & \multirow{3}{*}{ MSOR } & IT & 38 & 82 & 296 & - \\
\hline & & CPU & 0.0469 & 0.0781 & 0.3438 & - \\
\hline & & RES & $8.648 \times 10^{-6}$ & $8.408 \times 10^{-6}$ & $9.475 \times 10^{-6}$ & - \\
\hline \multirow{6}{*}{$m=30$} & \multirow{3}{*}{ RMSOR } & IT & 50 & 47 & 54 & 107 \\
\hline & & CPU & 0.0781 & 0.0675 & 0.0938 & 0.25 \\
\hline & & RES & $6.121 \times 10^{-6}$ & $1.691 \times 10^{-6}$ & $6.982 \times 10^{-6}$ & $8.419 \times 10^{-6}$ \\
\hline & \multirow{3}{*}{ MSOR } & IT & 57 & 108 & 410 & - \\
\hline & & CPU & 0.0938 & 0.2344 & 0.5781 & - \\
\hline & & RES & $3.915 \times 10^{-6}$ & $7.102 \times 10^{-6}$ & $9.266 \times 10^{-6}$ & - \\
\hline \multirow{6}{*}{$m=40$} & \multirow{3}{*}{ RMSOR } & IT & 65 & 62 & 74 & 129 \\
\hline & & $\mathrm{CPU}$ & 0.1406 & 0.1250 & 0.2188 & 0.3594 \\
\hline & & RES & $9.235 \times 10^{-6}$ & $7.330 \times 10^{-6}$ & $6.697 \times 10^{-6}$ & $9.493 \times 10^{-6}$ \\
\hline & \multirow{3}{*}{ MSOR } & IT & 80 & 138 & - & - \\
\hline & & $\mathrm{CPU}$ & 0.1875 & 0.2813 & - & - \\
\hline & & RES & $7.557 \times 10^{-6}$ & $8.111 \times 10^{-6}$ & - & - \\
\hline \multirow{6}{*}{$m=50$} & \multirow{3}{*}{ RMSOR } & IT & 79 & 76 & 91 & 156 \\
\hline & & $\mathrm{CPU}$ & 0.1719 & 0.1563 & 0.2031 & 0.3438 \\
\hline & & RES & $9.926 \times 10^{-6}$ & $8.954 \times 10^{-6}$ & $9.862 \times 10^{-6}$ & $8.250 \times 10^{-6}$ \\
\hline & \multirow{3}{*}{ MSOR } & IT & 100 & 174 & - & - \\
\hline & & $\mathrm{CPU}$ & 0.2188 & 0.3281 & - & - \\
\hline & & RES & $9.408 \times 10^{-6}$ & $8.764 \times 10^{-6}$ & - & - \\
\hline \multirow{6}{*}{$m=60$} & \multirow{3}{*}{ RMSOR } & IT & 93 & 89 & 110 & 178 \\
\hline & & CPU & 0.2969 & 0.2813 & 0.3906 & 0.5625 \\
\hline & & RES & $7.478 \times 10^{-6}$ & $9.642 \times 10^{-6}$ & $9.490 \times 10^{-6}$ & $8.071 \times 10^{-6}$ \\
\hline & \multirow{3}{*}{ MSOR } & IT & 119 & 211 & - & - \\
\hline & & CPU & 0.3281 & 0.5938 & - & - \\
\hline & & RES & $8.510 \times 10^{-6}$ & $7.022 \times 10^{-6}$ & - & - \\
\hline
\end{tabular}




\section{Conclusions}

In this paper, by introducing a relaxed matrix to obtain a new equivalent fixed-point form of the $\operatorname{LCP}(q, A)$, we establish a class of relaxed modulus-based matrix splitting (RMMS) iteration methods. Some sufficient conditions are presented to guarantee the convergence of RMMS iteration methods. Numerical examples show that the RMMS iteration method is feasible and overmatches the classical MMS iteration method under certain conditions.

It is noted that our approach can be extended to other modulus-based matrix splitting methods, such as two-step modulus-based matrix splitting iteration methods, accelerated modulus-based matrix splitting methods, and so on.

\section{Discussion}

From our numerical experiments, we find that both the MMS iteration method and the RMMS iteration method are sensitive to the iteration parameter. This implies that the iteration parameter may play an important part in these two methods. Therefore, the determination of the optimal parameters for these two methods could be still an open problem, which is an interesting topic in the future.

Author Contributions: Conceptualization, methodology, software S.W.; original draft preparation, C.L.; data curation, P.A.; guidance, review and revision, P.A.; translation, editing and review, S.W.; validation, S.W. All authors have read and agreed to the published version of the manuscript.

Funding: This research was supported by National Natural Science Foundation of China (No.11961082).

Institutional Review Board Statement: Not applicable.

Informed Consent Statement: Not applicable.

Data Availability Statement: Not applicable.

Acknowledgments: The authors would like to thank two anonymous referees for providing helpful suggestions, which greatly improved the paper.

Conflicts of Interest: The authors declare no conflict of interest.

\section{References}

1. Cottle, R.W.; Pang, J.-S.; Stone, R.E. The Linear Complementarity Problem; Academic: San Diego, CA, USA, 1992.

2. Murty, K.G. Linear Complementarity, Linear and Nonlinear Programming; Heldermann: Berlin, Germany, 1988.

3. Cottle, R.W.; Dantzig, G.B. Complementary pivot theory of mathematical programming. Linear Algebra Appl. $1968,1,103-125$. [CrossRef]

4. Schäfer, U. A linear complementarity problem with a P-matrix. SIAM Rev. 2004, 46, 189-201. [CrossRef]

5. Zheng, N.; Hayami, K.; Yin, J.-F. Modulus-type inner outer iteration methods for nonnegative constrained least squares problems. SIAM J. Matrix Anal. Appl. 2016, 37, 1250-1278. [CrossRef]

6. Ahn, B.H. Solutions of nonsymmetric linear complementarity problems by iterative methods. J. Optim. Theory Appl. 1981, 33, 175-185. [CrossRef]

7. Cryer, C.W. The solution of a quadratic programming using systematic overrelaxation. SIAM J. Control. 1971, 9, 385-392. [CrossRef]

8. Kappel, N.W.; Watson, L.T. Iterative algorithms for the linear complementarity problems. Int. J. Comput. Math. 1986, 19, $273-297$. [CrossRef]

9. Mangasarian, O.L. Solutions of symmetric linear complementarity problems by iterative methods. J. Optim. Theory Appl. 1977, 22, 465-485. [CrossRef]

10. Pang, J.-S. Necessary and sufficient conditions for the convergence of iterativemethods for the linear complementarity problem. J. Optim. Theory Appl. 1984, 42, 1-17. [CrossRef]

11. Tseng, P. On linear convergence of iterative methods for the variational inequality problem. J. Comput. Appl. Math. 1995, 60, 237-252. [CrossRef]

12. Bai, Z.-Z. On the convergence of the multisplitting methods for the linear complementarity problem. SIAM J. Matrix Anal. Appl. 1999, 21, 67-78. [CrossRef]

13. Bai, Z.-Z.; Evans, D. Matrix multisplitting methods with applications to linear complementarity problems: Parallel asynchronous methods. Int. J. Comput. Math. 2002, 79, 205-232. [CrossRef]

14. Bai, Z.-Z. Modulus-based matrix splitting iteration methods for linear complementarity problems. Numer. Linear Algebra Appl. 2010, 17, 917-933. [CrossRef] 
15. Van Bokhoven, W.M.G. A Class of Linear Complementarity Problems is Solvable in Polynomial Time; Unpublished Paper; Dept. of Electrical Engineering, University of Technology: Delft, The Netherlands, 1980.

16. Dong, J.-L.; Jiang, M.-Q. A modified modulus method for symmetric positive-definite linear complementarity problems. Numer. Linear Algebra Appl. 2009, 16, 129-143. [CrossRef]

17. Hadjidimos, A.; Tzoumas, M. Nonstationary extrapolated modulus algorithms for the solution of the linear complementarity problem. Linear Algebra Appl. 2009, 431, 197-210. [CrossRef]

18. Bai, Z.-Z.; Zhang, L.-L. Modulus-based synchronous multisplitting iteration methods for linear complementarity problems. Numer. Linear Algebra Appl. 2013, 20, 425-439. [CrossRef]

19. Bai, Z.-Z.; Zhang, L.-L. Modulus-based synchronous two-stage multisplitting iteration methods for linear complementarity problems. Numer. Algor. 2013, 62, 59-77. [CrossRef]

20. Zheng, N.; Yin, J.-F. Accelerated modulus-based matrix splitting iteration methods for linear complementarity problems. Numer. Algor. 2013, 64, 245-262. [CrossRef]

21. Zhang, L.-L. Two-step modulus-based matrix splitting iteration method for linear complementarity problems. Numer. Algor. 2011, 57, 83-99. [CrossRef]

22. $\mathrm{Wu}, \mathrm{S}$.-L.; Li, C.-X. Two-sweep modulus-based matrix splitting iteration methods for linear complementarity problems. J. Comput. Math. 2016, 302, 327-339. [CrossRef]

23. Li, W. A general modulus-based matrix splitting method for linear complementarity problems of H-matrices. Appl. Math. Lett. 2013, 26, 1159-1164. [CrossRef]

24. Hadjidimos, A.; Lapidakis, M.; Tzoumas, M. On iterative solution for linear complementarity problem with an $H_{+}$-matrix. SIAM J. Matrix Anal. Appl. 2011, 33, 97-110. [CrossRef]

25. Ma, C.-F.; Huang, N. Modified modulus-based matrix splitting algorithms for a class of weakly nondifferentiable nonlinear complementarity problems. Appl. Numer. Math. 2016, 108, 116-124. [CrossRef]

26. Xia, Z.-C.; Li, C.-L. Modulus-based matrix splitting iteration methods for a class of nonlinear complementarity problem. Appl. Math. Comput. 2015, 271, 34-42. [CrossRef]

27. Xie, S.-L.; Xu, H.-R.; Zeng, J.-P. Two-step modulus-based matrix splitting iteration method for a class of nonlinear complementarity problems. Linear Algebra Appl. 2016, 494, 1-10. [CrossRef]

28. Huang, N.; Ma, C.-F. The modulus-based matrix splitting algorithms for a class of weakly nondifferentiable nonlinear complementarity problems. Numer. Linear Algebra Appl. 2016, 23, 558-569. [CrossRef]

29. Hong, J.-T.; Li, C.-L. Modulus-based matrix splitting iteration methods for a class of implicit complementarity problems. Numer. Linear Algebra Appl. 2016, 23, 629-641. [CrossRef]

30. Wu, S.-L.; Guo, P. Modulus-based matrix splitting algorithms for the quasi-complementarity problems. Appl. Numer. Math. 2018, 132, 127-137 [CrossRef]

31. Mezzadri, F.; Galligani, E. Modulus-based matrix splitting methods for horizontal linear complementarity problems. Numer. Algor. 2020, 83, 201-219. [CrossRef]

32. Zheng, N.; Yin, J.-F. Convergence of accelerated modulus-based matrix splitting iteration methods for linear complementarity problem with an $H_{+}$-matrix. J. Comput. Appl. Math. 2014, 260, 281-293. [CrossRef]

33. Varga, R.S. Matrix Iterative Analysis; Prentice-Hall: Englewood Cliffs, NJ, USA, 1962.

34. Berman, A.; Plemmons, R.J. Nonnegative Matrices in the Mathematical Sciences; Academic: New York, NY, USA, 1979.

35. Frommer, A.; Mayer, G. Convergence of relaxed parallel multisplitting methods. Linear Algebra Appl. 1989, 119, 141-152. [CrossRef]

36. $\mathrm{Xu}, \mathrm{W} .-\mathrm{W}$. Modified modulus-based matrix splitting iteration methods for linear complementarity problems. Numer. Linear Algebra Appl. 2015, 22, 748-760. [CrossRef]

37. Zhang, L.-L.; Ren, Z.-R. Improved convergence theorems of modulus-based matrix splitting iteration methods for linear complementarity problems. Appl. Math. Lett. 2013, 26, 638-642. [CrossRef] 